\section{P100 IHC TESTING FOR SEBACEOUS NEOPLASMS: A RAPID REVIEW OF SCREENING ACCURACY AND APPLICATION OF GRADE}

J Natoli, M Alvarado. Kaiser Permanente (Southern California), Pasadena, CA, United States

\section{0:1136/bmjqs-2013-002293.163}

Background Lynch syndrome is the most common form of inherited colorectal cancer ( $\sim 3 \%$ of cases). Among patients with colorectal cancer tumours, immunohistochemistry (IHC) for mismatch repair proteins has demonstrated validity and utility as a screening test for Lynch syndrome, although guidelines differ on whether screening should be limited to patients at increased risk. IHC can be performed on other Lynch-related tumours, including sebaceous neoplasms, although it is unclear if IHC should be part of routine pathologic evaluation for sebaceous neoplasms.

Objectives To determine the clinical validity and utility of routine IHC testing of sebaceous neoplasms to inform development of guidance on screening for the Muir-Torre variant of Lynch syndrome.

Methods An AHRQ-based analytic framework was created. We conducted comprehensive searches to identify clinical studies that evaluated IHC testing of sebaceous neoplasms as a method of screening for Muir-Torre syndrome. GRADE was used for critical appraisal.

Results The body of evidence included 14 clinically heterogeneous studies representing approximately 300 patients. The weighted mean screen positive rate was $37 \%$. Few studies reported measures of clinical validity, although half of the studies reported the prevalence of visceral malignancy and/or results of germline mutation testing, suggesting possible reporting bias.

Discussion The overall body of evidence is of low quality and does not provide conclusive evidence for or against IHC testing for sebaceous neoplasms, either as a routine protocol or only among patients at high risk for Muir-Torre syndrome.

Implications for Guideline Developers/Users Rapid evidence reviews are useful for informing guidance development on rare/ genetic conditions.

\section{P107 DEVELOPING A CUSTOMISED WEB-BASED DATA EXTRACTION TOOL USING AN EXISTING CUSTOMER RELATIONSHIP SERVICE: THINKING OUTSIDE THE BOX}

${ }^{1,2}$ E Nagler, ${ }^{3}$ V Liébaut, ${ }^{2,4} \mathrm{M}$ Haller, ${ }^{2,5} \mathrm{~S}$ van der Veer, ${ }^{2,6} \mathrm{D}$ Bolignano, ${ }^{2,7} \mathrm{I}$ Nistor, ${ }^{1,2} \mathrm{~W}$ Van
Biesen. ${ }^{1}$ Renal Division, Department of Internal Medicine, Ghent University Hospital,
Ghent, Belgium; ${ }^{2}$ ERBP, Ghent University Hospital, Ghent, Belgium; ${ }^{3} 4 C$ Consulting,
Mechelen, Belgium; ${ }^{4}$ Section for Clinical Biometrics, Medical University of Vienna,
Vienna, Austria; ${ }^{5}$ Department of Medical Informatics, Academic Medical Center,
Amsterdam, The Netherlands; ${ }^{6}$ CNR-IBIM Reggio Calabria, Italy; ${ }^{7}$ Nephrology
Department, GT. Popa University of Medicine and Pharmacy, Lasi, Romania

10:1136/bmjqs-2013-002293.164

Background Standardised duplicate data extraction and tabulation can be challenging for organisations that develop multiple guidelines involving multiple and remote systematic review teams simultaneously.

Objectives To develop a web-based tool for facilitating duplicate data extraction and efficient discrepancy resolution.

Methods Based on previous experiences with word-processing and spreadsheet tools, European Renal Best Practice listed their system requirements and collaborated with a consultancy company to identify appropriate customisable software.
Results We wanted the system to: be web-based, guide reviewers through a standardised data extraction form, be easy-to-use and manage, allow enough flexibility to accommodate different guideline topics, be free-of-charge and easily accessible from different locations without the need for downloading software. We identified a customer relationship management service, Salesforce, that allowed us to build a data extraction module using their backbone structure. It incorporates centralised management of multiple systematic reviews simultaneously, batch allocation of studies to individual reviewers, guided customised point-andclick data extraction, generation of tables to assist discrepancy resolution with easy export to a cvs-file extension format.

Discussion This project represents a continuous effort to facilitate efficient and high-quality systematic reviewing with participation of our guideline development groups throughout the systematic reviewing process. A first version of the system is currently being evaluated.

Implications for Guideline Developers/Users Customising existing software for guideline development purposes might be an attractive and inexpensive alternative to developing new tools for data extraction when full participation of the guideline development group in the systematic review process is desired.

\section{P108 PUBLICATIONS IMPROVE WOUND CARE GUIDELINE QUALITY AND IMPACT}

${ }^{1} \mathrm{~L}$ Bolton, ${ }^{2} \mathrm{~S}$ Girolami, ${ }^{3} \mathrm{~L}$ Corbett, ${ }^{4} \mathrm{D}$ Merkle. ${ }^{1}$ Robert Wood Johnson University Medical School, New Brunswick, USA; ${ }^{2}$ Therapy Support, Cincinnati, OH, USA; ${ }^{3}$ Hartford Hospital, Hartford, CT, USA; ${ }^{4}$ Bridgeport Hospital, Bridgeport, CT, USA

\section{0:1136/bmjqs-2013-002293.165}

Background High quality evidence based (EB) wound care guidelines reduce costs of care and improve outcomes. They may help stem the growing burden of chronic wound care only if disseminated and used.

Context Publications supported development, quality and dissemination of EB wound care guidelines with content-validity (CV).

Description of Best Practice Multidisciplinary volunteer guideline developers from a global, non-profit wound care organisation with patient and wound-related specialty membership, used standardised evidence strength criteria and independently-rated $\mathrm{CV}$ Index as standardised recommendation strength to develop inclusive venous and pressure ulcer "Guidelines of Guidelines". Representatives of six North American societies collaboratively published research and educational opportunities identified by this unique guideline development process.

Lessons for Guideline Developers, Adapters, Implementers, and/ or Users Publications accessible at the organisation website transparently described the guideline development process, a hallmark of guideline quality while enhancing awareness of the guidelines and EB CV recommendations worth implementing. Publications in multidisciplinary journals minimised sampling bias by inviting readership to complete online CV surveys. Publishing research and educational opportunities focused the field on $\mathrm{EB}$ research and education needs and fostered international cooperation, helping unify wound care across countries, settings and specialties. Medical school and university nursing and physical therapy programmes are disseminating core messages of EB $\mathrm{CV}$ wound care by using the guidelines, publications, patient brochures, algorithms and implementation tools to improve quality and consistency of care.

Conclusion Publications enhanced venous and pressure ulcer guideline development quality, awareness and use while unifying 
wound care and highlighting needed research and education initiatives.

\section{P111 A WORKFLOW CHECKLIST FOR IMPROVING MANAGEMENT OF THE GUIDELINE DEVELOPMENT PROCESS}

${ }^{1,2} \mathrm{M}$ Haller, ${ }^{1,3} \mathrm{E}$ Nagler, ${ }^{1,4} \mathrm{~S}$ van der Veer, ${ }^{1,5}$ I Nistor, ${ }^{1,6} \mathrm{D}$ Bolignano, ${ }^{1,3} \mathrm{~W}$ Van Biesen. ${ }^{1}$ European Renal Best Practice, Ghent University Hospital, Ghent, Belgium; ${ }^{2}$ Center for Medical Statistics (CeMSIIS), Medical University Vienna, Vienna, Austria; ${ }^{3}$ Renal Division, Department of Internal Medicine, Ghent University Hospital, Ghent, Belgium; ${ }^{4}$ Academic Medical Center, Department of Medical Informatics, Amsterdam, The Netherlands; ${ }^{5}$ Nephrology Department, GT. Popa University of Medicine and Pharmacy, Lasi, Romania; ${ }^{6}$ CNR-IBIM, Clinical Epidemiology and Physiopathology of Renal Diseases Reggio, Calabria, Italy

\section{0:1136/bmjqs-2013-002293.166}

Background Keeping track of progress and meeting deadlines can be difficult for organisations that simultaneously develop multiple guidelines involving multiple systematic review teams.

Objectives To improve planning, organising and managing of guideline development projects by creating a standardised workflow checklist.

Methods In a one-day meeting, the five guideline development methodologists, the chairman and the editorial assistant identified the main steps involved in the guideline development process. For each step, we identified specific tasks and ordered them chronologically. All decisions were made based on group consensus. The identified steps and tasks formed the basic elements of the workflow checklist.

Results We identified the need for two separate checklists per guideline development project; one for overall workflow and one for each clinical question covered by the guideline. The overall guideline development workflow checklist comprised 42 tasks organised in 11 sequential steps, including items such as topic selection, composition of the guideline development group, and framing the questions. For each clinical question we identified 27 tasks organised in 8 sequential steps, excluding steps already covered by the overall workflow.

Discussion This workflow checklist represents a first step in developing a standardised project management strategy to improve efficient management of the guideline development process. Further development of this tool involves selecting appropriate software for practical implementation applicable not only for our own means but also for those of other groups.

Implications for Guideline Developers/Users We believe developing a standardised workflow checklist will improve efficient management of guideline development and allows transparent and up-to-date communication of its progress.

\section{P114 THE QUALITY OF EVIDENCE OF SYSTEMATIC REVIEWS OF TRADITIONAL CHINESE MEDICINE: A CROSS- SECTIONAL STUDY}

${ }^{1,2} \mathrm{Y}$ Chen, ${ }^{1,2} \mathrm{Q}$ Wu, ${ }^{1,2} \mathrm{X}$ Wang, ${ }^{1,2} \mathrm{Q}$ Wang, ${ }^{1,2} \mathrm{D}$ Wei, ${ }^{1,2} \mathrm{~L}$ Yao,${ }^{3} \mathrm{~F}$ Liang, ${ }^{1,2}, \mathrm{M}$ Wang, ${ }^{1,2} \mathrm{~K}$ Yang. 'Evidence-Based Medicine Center of Lanzhou University, Lanzhou, China; ${ }^{2}$ Chinese GRADE Center, Lanzhou, China; ${ }^{3}$ The First Hospital of Lanzhou University, Lanzhou, China

\section{0:1136/bmjqs-2013-002293.167}

Background There has been an increasing interest in systematic reviews of traditional Chinese medicine (SR-TCM) over the past
10 years. Little is known about the quality of evidence of SRTCM.

Methods GRADE (Grading of Recommendations Assessment, Development and Evaluation) system is a tool to rate evidence quality of SRs and other evidence body. We searched CBM (China Biomedicine Database) from 1978 to 2012 and included all SR-TCM in the field of cancer treatment. We used GRADE system to assess the quality of evidence of those SRs. Two reviewers independently screened the titles and abstracts of identified studies. Full texts of potentially included articles were further assessed. Disagreements were resolved by discussion.

Results The preliminary results showed that the quality of evidence of SR-TCM were: high (1\%), moderate (25\%), low $(50 \%)$, very low (24\%). We also compared with the quality of evidence of SRs published in Chinese medical journals $(5 \%$, 27\%, 49\%, 19\%) and Cochrane SRs (5\%, 27\%, 49\%, 19\%). Risk of bias, inconsistency and publication bias were the major factors for downgrading evidence of SR-TCM.

Conclusion More and more SR-TCM had been published in Chinese medical journals, however, the proportion of high quality evidence is lower and the very low quality evidence is higher compared with national and international levels.

\section{P115 HOW MANY CLUSTER RANDOMIZED CONTROLLED TRIALS WERE USED IN CLINICAL PRACTICE GUIDELINES?}

${ }^{1,2} \mathrm{Y}$ Chen, ${ }^{1,2} \mathrm{Q}$ Wu, ${ }^{1,2} \mathrm{X}$ Wang, ${ }^{1,2} \mathrm{Q}$ Wang, ${ }^{1,2} \mathrm{D}$ Wei, ${ }^{1,2} \mathrm{~L}$ Yao, ${ }^{1,2} \mathrm{~F}$ Liang, ${ }^{3} \mathrm{M}$ Wang, ${ }^{1,2} \mathrm{~K}$ Yang. 'Evidence-Based Medicine Center of Lanzhou University, Lanzhou, China; ${ }^{2}$ Chinese GRADE Center, Lanzhou, China; ${ }^{3}$ The First Hospital of Lanzhou University, Lanzhou, China

\section{0:1136/bmjqs-2013-002293.168}

Background There has been an increasing interest in cluster randomised controlled trial (CRT) over the past 20 years. Little is known about how many CRTs were used by clinical practice guidelines.

Methods We searched National Guideline Clearinghouse (NGC) which is a public resource for evidence-based clinical practice guidelines on December 31, 2012. We selected guidelines which provide MEDLINE full-text linking and then we checked all references cited by those guidelines.

Results We included 564 guidelines and they contained 57495 references. We identified 13 guidelines cited 17 cluster randomised controlled trials as their references. Guidelines are about primary care, cancer, obesity, breastfeeding, cardiovascular and orthopaedics disease. Screening studies accounts for as much as $35 \%$ (6) of all CRTs.

Conclusion Cluster randomised controlled trial is considered as the golden standard to assess the effect of intervention in health research. Based on the retrieval strategy study for cluster randomised controlled trial we developed, we estimated that there are about 8000 CRTs in Medline, however, only 17 CRTs were used or cited by clinical practice guidelines, the reasons of low utilisation of CRTs in guidelines are now being investigated and we are going to present the final findings.

\section{P117 UNIFORMITY IN ANAESTHESIOLOGY RECOMMENDATIONS}

${ }^{1}$ I Loman, ${ }^{2} \mathrm{~A}$ Schuurhuis. 'Knowledge Institute of Medical Specialists, Utrecht, The Netherlands; ${ }^{2}$ The Netherlands Society of Anesthesiologists, Utrecht, The Netherlands

10:1136/bmjqs-2013-002293.169 